\title{
Role of Double-contrast Barium Enema in Crohn's Colitis
}

\author{
Uma Debi ${ }^{1}$, Lokesh Singh ${ }^{2}$, Vaibhav Gulia ${ }^{3}$, Vishal Sharma ${ }^{4}$, Rohan S Kamat ${ }^{5}$, Richa S Chauhan ${ }^{6}$, Kaushal K Prasad $^{7}$
}

\begin{abstract}
Background: Double-contrast barium enema (DCBE) is a safe, noninvasive, inexpensive, and cost-effective imaging technique. It offers complete visualization of the colonic mucosa. Inflammatory bowel disease (IBD) including Crohn's disease can easily be diagnosed using the DCBE technique. Aims and objectives: To demonstrate the accuracy of DCBE in diagnosis of Crohn's colitis.

Materials and methods: A retrospective review of DCBE examination in 57 patients of colonic Crohn's disease was performed. A barium suspension of medium viscosity at $100 \% \mathrm{w} / \mathrm{v}$ was used. Each DCBE was evaluated by two experienced gastrointestinal radiologists independently without any knowledge of the colonoscopic findings.

Results: In $89 \%$ of patients, the radiological diagnosis corresponded with the clinical diagnosis of Crohn's disease. Five patients were misclassified as tubercular and one as ulcerative colitis. Asymmetric disease with rectal sparing and discrete ulcers were the most commonly observed radiological findings. About $19 \%$ of patients showed the features of early Crohn's as suggested by the finding of aphthoid ulcerations with background of the normal mucosa. The left-sided colon was more commonly involved compared with the right side with the transverse colon being the most commonly involved part.

Conclusion: The double-contrast examination technique, because of its high accuracy in diagnosing Crohn's disease and due to its relatively specific signs, is suggested to be the preferred examination in the radiological assessment of inflammatory bowel disease.

Keywords: Barium enema, Colitis, Colonoscopy, Crohn's.

Journal of Postgraduate Medicine, Education and Research (2020): 10.5005/jp-journals-10028-1354
\end{abstract}

\section{INTRODUCTION}

Any region of the gastrointestinal tract from the mouth to the anus can be involved by Crohn's disease. More than half of the patients, usually, present with small bowel disease, with or without the involvement of the colon. However, in approximately onefourth of the patients, the disease is confined to the large bowel. The single-contrast barium enema examination has long been a proven method of visualization of the colon for the assessment of inflammatory diseases; however, the double-contrast barium enema (DCBE) examination offers more complete visualization of the colonic mucosa. Several investigators have reported that the double-contrast method shows considerable improvement over the single-contrast technique in the sensitivity of detection of disease. ${ }^{1-3}$ In a retrospective analytical study by Margulis et al, conventional barium enema was observed to show less accuracy, with merely $79 \%$ of patients with ulcerative colitis and $70 \%$ with colonic Crohn's disease being accurately diagnosed. ${ }^{4}$ Lately, Laufer et al. have recommended DCBE for detecting the early changes of colonic Crohn's disease and ulcerative colitis. ${ }^{5-8}$ In this article, we report our experience with the DCBE technique and its accuracy in evaluating the colonic Crohn's disease.

\section{Aims and Objectives}

To demonstrate the accuracy of DCBE in diagnosis of Crohn's colitis.

\section{Materials and Methods}

The use of DCBE in 57 consecutive documented cases of Crohn's disease of the colon was retrospectively analyzed. Of all the cases, 24 were females (17-61 years) and 33 were males (9-65 years). To be included in the study, all quiescent cases were required to have had previous active disease documented either endoscopically or radiographically at this institution. All the patients were asked for
1-3,5,6 Department of Radiodiagnosis and Imaging, Postgraduate Institute of Medical Education and Research, Chandigarh, India

4,7Department of Gastroenterology, Postgraduate Institute of Medical Education and Research, Chandigarh, India

Corresponding Author: Uma Debi, Department of Radiodiagnosis and Imaging, Postgraduate Institute of Medical Education and Research, Chandigarh, India, Phone: +91 2147483647, e-mail: debi_uma@yahoo. co.in

How to cite this article: Debi U, Singh L, Gulia V, et al. Role of Doublecontrast Barium Enema in Crohn's Colitis. J Postgrad Med Edu Res 2020;54(2):40-44.

Source of support: Nil

Conflict of interest: None

the bowel preparation prior to the examination, which comprised of a clear liquid diet for $\mathbf{2 4}$ hours, one or two sets of laxatives the day before, and cleansing enemas. A barium suspension of medium viscosity at $100 \% \mathrm{w} / \mathrm{v}$ was used as the positive oral contrast.

Each DCBE was evaluated by two experienced gastrointestinal radiologists independently without any knowledge of the colonoscopic findings. When there was disagreement in classification among the radiologists, films were reviewed together and a consensus was achieved. Classification was made by using the following criteria: aphthous ulcers or deep ulcerations (or both) with an asymmetric and discontinuous distribution indicated granulomatous colitis. The presence of polypoid changes, fistulas, and luminal shortening and narrowing was also considered for the classification of disease. All patients studied had colonoscopic examinations within 1 week before the DCBE. The reports of colonoscopy were prepared on the basis of retrospective correlation with the radiographic studies. The condition of each patient was evaluated by a gastroenterologist, who determined the final clinical 
diagnosis on the basis of an aggregate of findings acquired from all diagnostic methods (i.e., endoscopy, DCBE, pathologic analysis, and clinical presentation). This diagnosis was made after the final assessment of the sensitivity and specificity of the DCBE.

\section{Results}

Fifty-seven patients with a final clinical diagnosis of active granulomatous colitis were studied with DCBE. A total of 24 cases were females with mean age of 33.1 years and 33 were males with mean age of 31.5 years. Chronic diarrhea, weight loss, and abdominal pain were the most commonly observed clinical features. A total of 51 cases (89\%) were correctly identified as Crohn's colitis on DCBE. Five cases were wrongly diagnosed as tuberculosis and one was diagnosed as ulcerative colitis. The incidence of radiographic findings, clinical features, and the diagnostic accuracy is depicted in Tables 1 to 4.

Discrete and aphthous ulcers (Fig. 1) with rectal sparing, terminal ileal involvement (Fig. 2), narrowing (Figs 3 and 4), strictures

Table 1: Radiological features

\begin{tabular}{ll}
\hline Radiologic findings & Incidence $(n=57)$ \\
\hline Aphthous ulcers & $11(19.3 \%)$ \\
Discrete and deep ulcers & $27(47.4 \%)$ \\
Asymmetric/discontinuous disease & $56(98.2 \%)$ \\
Cobblestoning & $16(28.1 \%)$ \\
Terminal ileal involvement & $20(35.1 \%)$ \\
Rectal sparing & $56(98.2 \%)$ \\
Fistula and sinuses & $10(17.5 \%)$ \\
Narrowing and stricture & $29(50.8 \%)$ \\
Pseudodiverticula & $5(8.8 \%)$ \\
\hline
\end{tabular}

Table 2: Colon involvement

\begin{tabular}{ll}
\hline Part involved & Incidence $(n=57)$ \\
\hline Cecum & $12(21 \%)$ \\
Ascending colon & $11(19.3 \%)$ \\
Transverse colon & $19(33.3 \%)$ \\
Descending colon & $13(22.8 \%)$ \\
Sigmoid & $16(28.1 \%)$ \\
\hline
\end{tabular}

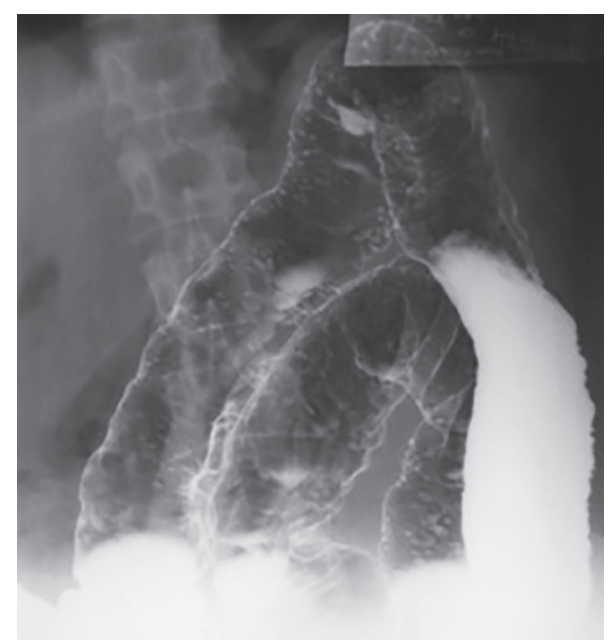

Fig. 1: Multiple aphthoid ulcers are seen with surrounding halo on background of normal mucosa
(Fig. 5) and pseudodiverticula formation (Fig. 6). Discontinuous disease (Fig. 5) formed the basis for diagnosis in most of the cases. Other less specific features were sinus tract (Fig. 7), fistula formation (Figs 8 and 9), double tract (Fig. 10) cobblestoning (Fig. 11) and transverse colon (Fig. 12) was the most commonly involved part with left sided disease being more commonly seen than the right side (Fig. 10).

\section{Discussion}

The double-contrast colon examination (DCE) is now widely recognized as demonstrating most changes associated with inflammatory disease more completely than the single-contrast study. ${ }^{2,7}$ The ability of the DCE to demonstrate the mucosal surface entirely makes the examination a formidable rival to endoscopy.

The features of Crohn's disease of the small bowel are typical. The incidence of small bowel involvement in Crohn's disease has been reported to be $80-100 \%$ in some of the studies while some reports have suggested it to be as low as $41-56 \%{ }^{2}$ The wide range of these incidences may contemplate the radiographic small bowel examinations to be false negative, as demonstrated in approximately $50-70 \%$ of cases who underwent histopathologic

Table 3: Clinical characteristics

\begin{tabular}{ll}
\hline Clinical features & Incidence $(n=57)$ \\
\hline Sex ratio (M:F) & $33: 24$ \\
Chronic diarrhea & $35(61.4 \%)$ \\
Fever & $11(19.3 \%)$ \\
Weight loss & $36(63.1 \%)$ \\
Hematochezia & $15(26.35 \%)$ \\
Intestinal obstruction & $11(19.3 \%)$ \\
Abdominal pain & $38(66.7 \%)$ \\
Abdominal lump & $9(15.8 \%)$ \\
Growth retardation & $7(12.3 \%)$ \\
Fistula-in-ano & $5(8.7 \%)$ \\
\hline
\end{tabular}

Table 4: Accuracy of barium enema

\begin{tabular}{llll}
\hline $\begin{array}{l}\text { Radiologic } \\
\text { diagnosis }\end{array}$ & Total patients & $\begin{array}{l}\text { Diagnosed on } \\
\text { barium enema }\end{array}$ & Accuracy \\
\hline Crohn's disease & 57 & 51 & $89.5 \%$ \\
\hline
\end{tabular}

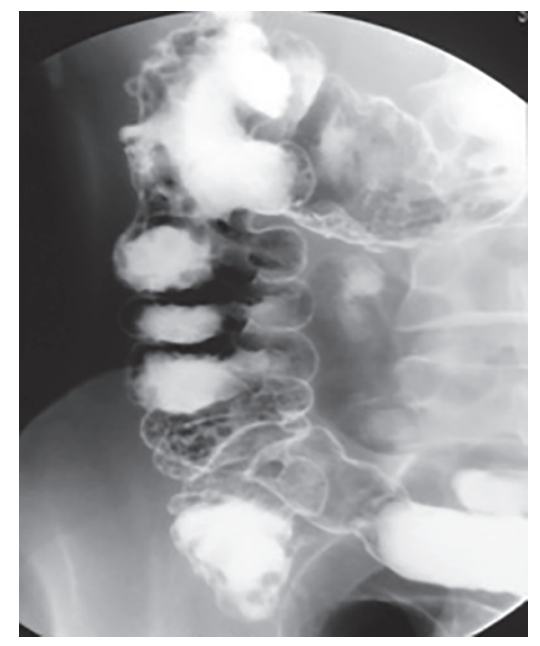

Fig. 2: Cecal and terminal ileal involvement is seen with multiple ulcerations and narrowing. To be differentiated from ileocecal TB 


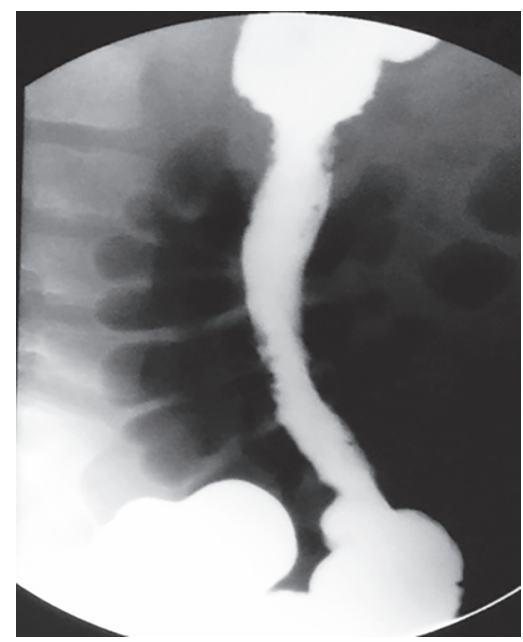

Fig. 3: Mild narrowing involving descending colon with ulcerations

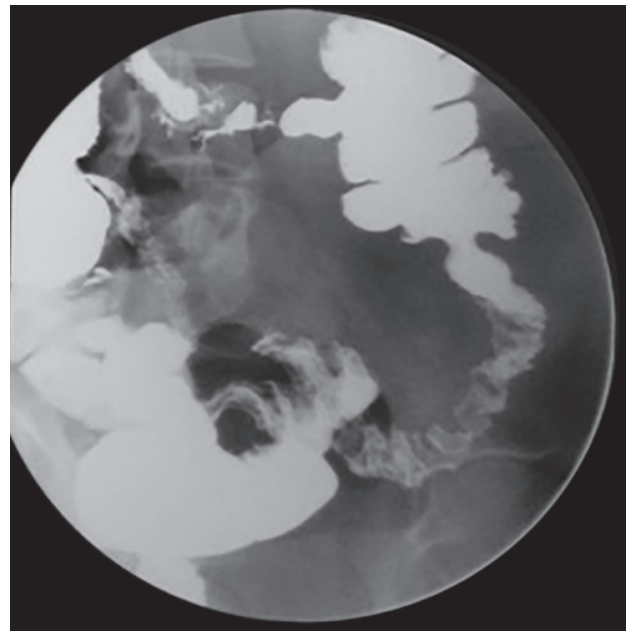

Fig. 5: Asymmetric and patchy involvement of large gut seen causing strictures, narrowing and ulceration in transverse colon and sigmoid
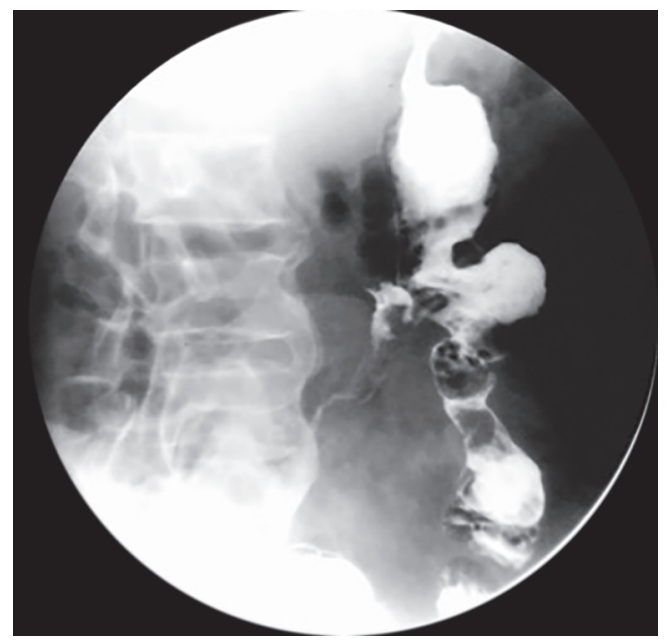

Fig. 7: Stricture descending colon with leakage of barium and pseudodiverticula formation

testing of the small bowel later on. Consequently, the recognition of the true frequency of the normal small bowel follow-through examinations in colonic Crohn's disease becomes necessary. In such patients, the early diagnosis necessitates the use of a radiographic

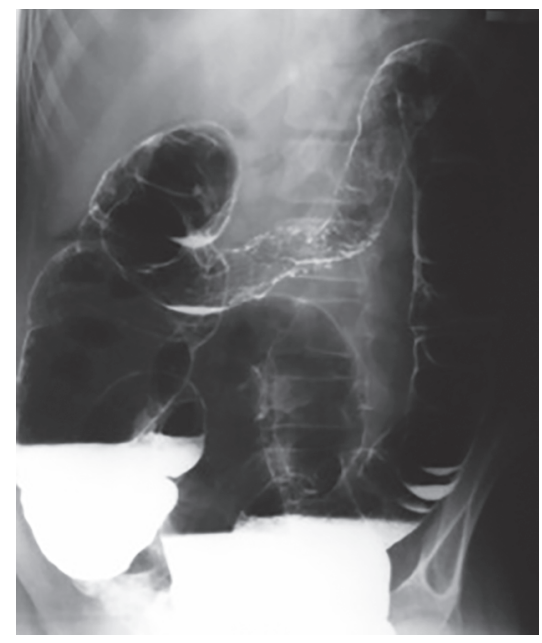

Fig. 4: Mild narrowing of transverse colon with multiple ulcerations

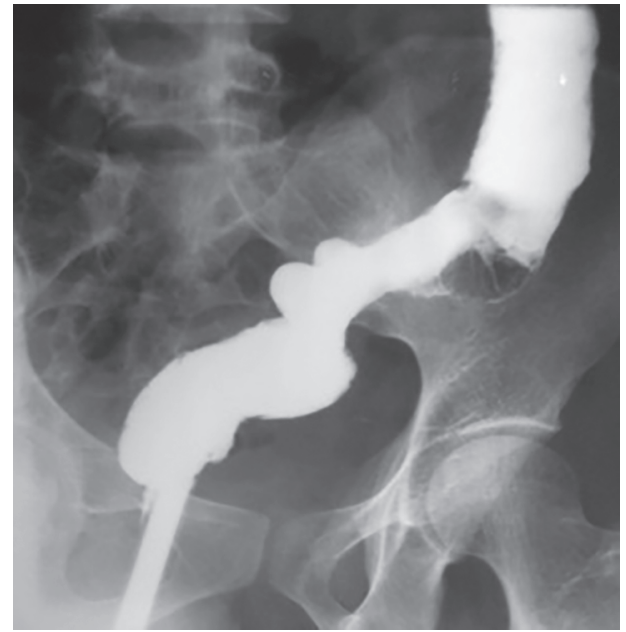

Fig. 6: Smooth narrowing of sigmoid colon with pseudodiverticula

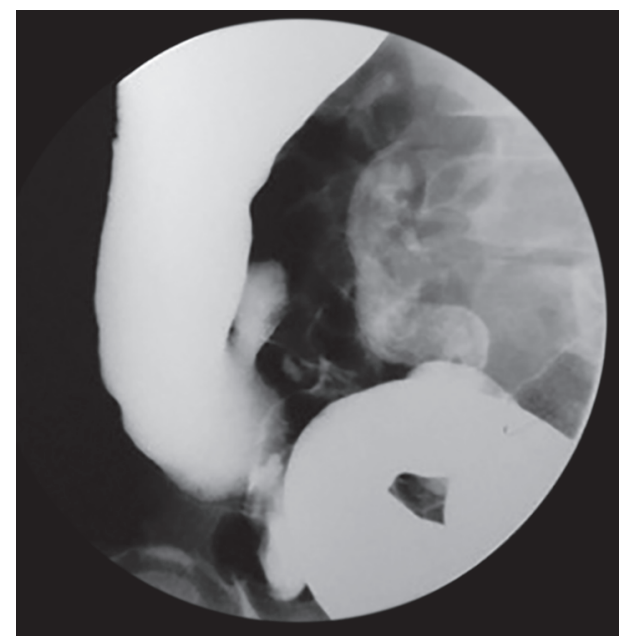

Fig. 8: Fistulous communication between sigmoid and ileal loops secondary to Crohn's colitis

examination of the colon that can reliably depict the fine mucosal changes.

The superficial mucosal ulcerations along with lymphoid hyperplasia have been described as the early lesions of the Crohn's 


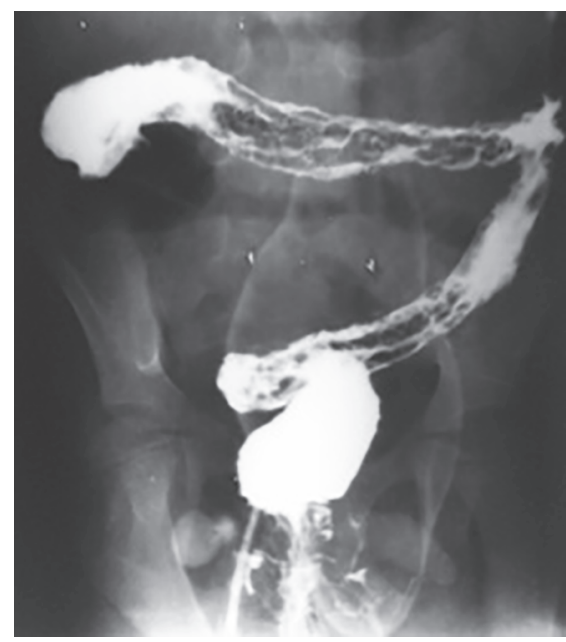

Fig. 9: Multiple perianal fistulas and sinuses

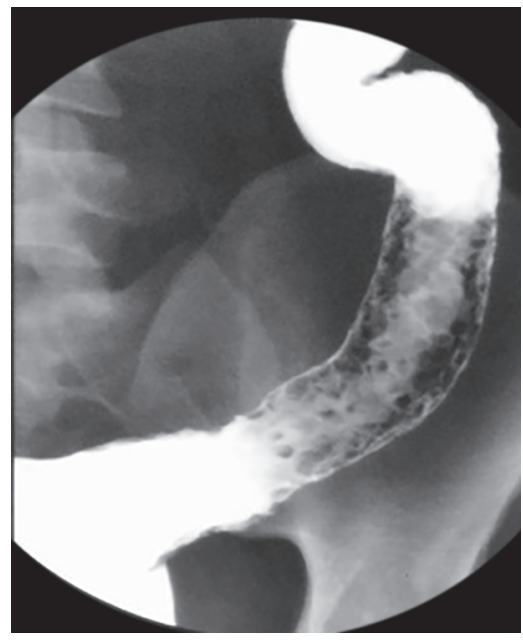

Fig. 11: Cobblestoning seen in sigmoid colon

disease by Morson and Dawson. The earliest pattern is usually the appearance of these lesions as small, aphthous ulcers. ${ }^{9}$ In approximately $50 \%$ patients with Crohn's colitis, these aphthous ulcers are found in isolation or in conjunction with areas of more severe disease. In our study, these aphthoid ulcers were detected in about $19 \%$ of patients. They are often encountered en face on DCE as well-defined, oval or round, variable-sized (from one to several millimeters) barium accumulations, with an encircling halo. ${ }^{2}$ Brahme and Fork observed the unstability of these lesions as they were shown to be either progressing to severe destruction or, less likely, regressing completely. ${ }^{10}$ The radiologic depiction of these aphthous ulcers in earlier phase of the disease has important clinical implications. In those patients who have only early lesions, this radiologic demonstration establishes the diagnosis and prevents further unnecessary investigations. It also allows earlier symptomatic treatment. In patients with colitis, the demonstration of these early lesions on the background of normal mucosa makes it easy to differentiate between granulomatous and ulcerative colitis. ${ }^{11}$ The target lesions, typical of Crohn's disease, are not observed in ulcerative colitis because the mucosa surrounding the ulcer is granular rather than flat.

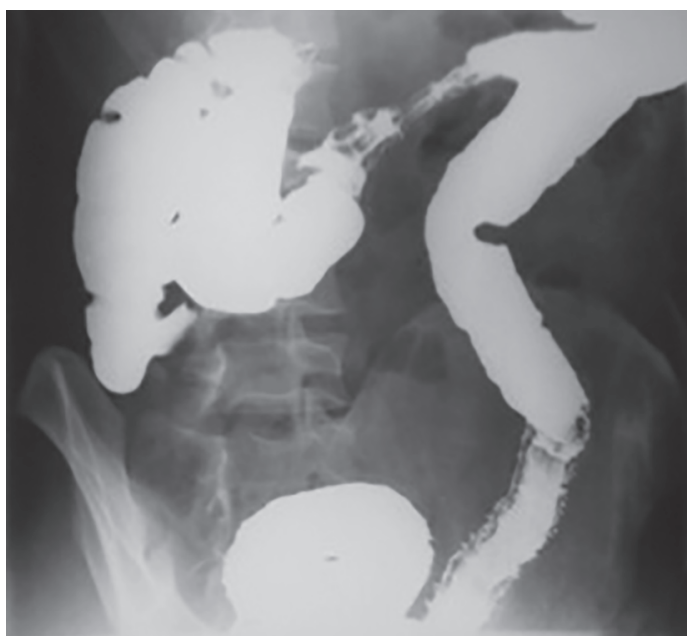

Fig. 10: Double tracting seen in sigmoid with ulceration and narrowing in transverse colon

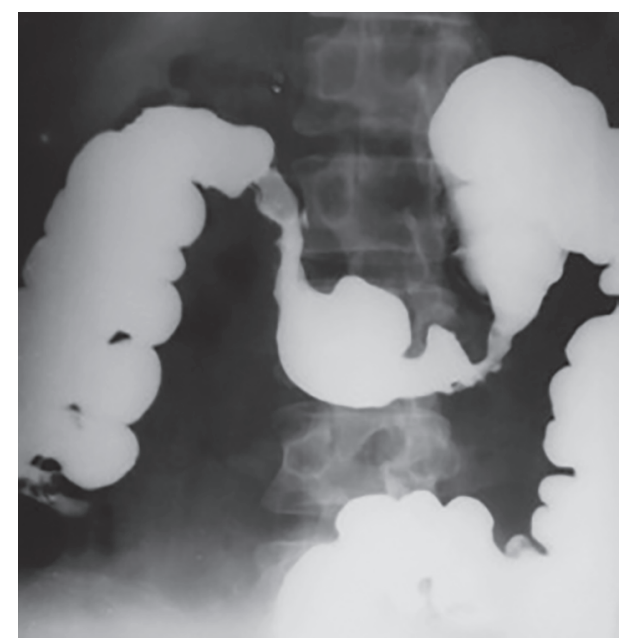

Fig. 12: Multiple strictures transverse colon with pseudodiverticula formation and ulcerations

The aphthoid ulcerations may regress, remain stable, or may enlarge and deepen. Adjacent ulcerations may merge taking the form of a meshwork of longitudinal ulcers and transverse fissures producing a cobblestone appearance. ${ }^{12}$ Cobblestoning was seen in about $28 \%$ of cases in our series.

The ulcer depth may be of significant diagnostic aid in inflammatory bowel disease. Deep ulcers $(>3 \mathrm{~mm}$ ) are frequently seen with Crohn's disease rather than in ulcerative colitis. ${ }^{2}$ We found deep ulcers in $47 \%$ of the cases in our study.

Sinuses and fistulas are a hallmark of Crohn's disease. Deep ulcers usually sink in beyond the submucosa with resultant formation of knife-shaped fistulas. ${ }^{13}$

A patchy distribution pattern is another hallmark of this disease entity. It may be seen as noncontiguous areas of involvement along the length of the bowel or as a localized asymmetric involvement of a single region. At times, this asymmetric disease pattern may result in the formation of pseudodiverticulae. All cases except for one showed a patchy distribution in our series with pseudodiverticula formation in about $9 \%$ cases.

The distribution pattern of the disease in the colon has been stressed upon in the literature. Margulis et al. observed that the 
confined right-sided colonic involvement commonly indicates Crohn's disease. ${ }^{4}$ As opposed to this observation, the limited involvement of the distal colon can be seen in both Crohn's disease and ulcerative colitis. However, barium enema usually shows the rectum to be normal in Crohn's disease. Rectal involvement, when occurs in Crohn's disease, is frequently evident in the form of discrete ulcers. ${ }^{2}$ In our series, left-sided involvement was seen more commonly.

Crohn's disease is an inflammatory condition that affects the bowel transmurally resulting into bowel wall thickening and fibrosis. This leads to luminal narrowing as well as foreshortening of the bowel. ${ }^{14,15}$

The barium enema examination (of any form) is contraindicated in the patients with the toxic megacolon. It is prudent to defer barium enema in patients of severe active colitis till the patient improvises clinically.

\section{Conclusion}

The DCBE is a safe investigation modality in diagnosing colonic Crohn's disease. Recognition of the disease at any stage is possible by depicting discrete and aphthoid ulcers, sinus, fistula with rectal sparing, as well as discontinuous disease and for that the technique of double-contrast examination should be employed. Because of its high accuracy and relatively specific signs, it is suggested to be the preferred examination in the radiological assessment of inflammatory bowel diseases.

\section{References}

1. Fennessy JJ, Sparberg M, Kirsner JB. Early roentgen manifestations of mild ulcerative colitis and proctitis. Radiology 1966;87(5):848-885. DOI: $10.1148 / 87.5 .848$.

2. Fraser GM, Findlay JM. The double contrast enema in ulcerative and Crohn's colitis. Clin Radiol 1976;27(1):103-112. DOI: 10.1016/S00099260(76)80030-X.
3. Kelvin FM, Oddson TA, Rice RP, et al. Double contrast barium enema in Crohn's disease and Ulcerative colitis. AJR Am J Roentgenol 1978;131(2):207-213. DOI: 10.2214/ajr.131.2.207.

4. Margulis AR, Goldberg HI, Lawson TL, et al. The overlapping spectrum of ulcerative and granulomatous colitis: a roentgenographic pathologic study. Am J Roentgenol 1971;113(2):325-334.DOI: 10.2214/ ajr.113.2.325.

5. Laufer I. The radiologic demonstration of early changes in ulcerative colitis by double contrast technique. J Can Assoc Radiol 1975;26(2):116-121.

6. Laufer I, Mullens JE, Hamilton J. Correlation of endoscopy and double contrast radiography in the early stages of ulcerative and granulomatous colitis. Radiology 1976;118(1):1-5. DOI: 10.1148/ 118.1.1.

7. Laufer I. Air contrast studies of the colon in inflammatory bowel disease. Crit Rev Diagn Imaging 1977;9(4):421-447.

8. Laufer I, Hamilton J. The radiological differentiation between ulcerative and granulomatous colitis by double contrast radiology. Am J Gastroenterol 1976;66(3):259-269.

9. Morson BC, Dawson IMP. Gastrointestinal Pathology. Philadelphia: Davis; 1972.

10. Brahme F, Fork FT. Dynamic aspects of Crohn's disease. Radiologe 1975;15(12):463-468.

11. Laufer I, Coustopolos L. Early lesions of Crohn's disease. Am J Roentgenol 1978;130(2):307-311. DOI: 10.2214/ajr.130.2.307.

12. Bartram Cl. Ulcerative Colitis. In: Bartram Cl, ed. Radiology in Inflammatory Bowel Disease. New York: Marcel Dekker; 1983. pp. 31-62.

13. Lichtenstein JE. Radiologic-pathologic correlation of inflammatory bowel disease. Radiol Clin North Am 1987;25(1):234.

14. Gore RM, Gaharemani GG, Miller FH. Inflammatory disease. In: Margulis AR, ed. Advances in Radiology of the Alimentary Tube. Heidelberg: Springer-Verlag; 1998. pp. 185-215.

15. Prince $A B$, Morson $B C$. Inflammatory bowel disease: the surgical pathology of Crohn's disease and ulcerative colitis. Hum Pathol 1987;6(1):7-29. DOI: 10.1016/S0046-8177(75) 80107-9. 